\title{
Avaliação da gestão de resíduos sólidos em municípios da região Centro Sul - Ceará
}

\author{
Evaluation of solid waste management in municipalities in the Center-South region - Ceará \\ Evaluación de la gestión de residuos sólidos en municipios de la región Centro-Sur - Ceará
}

Recebido: 19/10/2021 | Revisado: 28/10/2021 | Aceito: 03/11/2021 | Publicado: 05/11/2021

\author{
Valdenira Carlos da Silva \\ ORCID: https://orcid.org/0000-0002-5084-7248 \\ Universidade Federal do Cariri, Brasil \\ E-mail: valdenira.carlos@aluno.ufca.edu.br \\ Maria Gorethe de Sousa Lima Brito \\ ORCID: https://orcid.org/0000-0001-8977-1116 \\ Universidade Federal do Cariri, Brasil \\ E-mail: gorethe.lima@ufca.edu.br
}

\begin{abstract}
Resumo
O objetivo neste estudo é avaliar a condição da gestão integrada de resíduos sólidos em municípios da região do Centro-Sul do Ceará, por meio da utilização do Índice da Condição de Gestão de Resíduos Atualizado (ICGRA). Este índice foi desenvolvido com 62 indicadores dispostos em quatro itens: características do sistema, planejamento do sistema, condições operacionais e alinhamento da gestão à Política Nacional de Resíduos Sólidos e novas técnicas. O percurso metodológico foi realizado mediante aplicação da planilha do ICGRA a cada um dos municípios estudados (Iguatu, Jucás, Cariús, Saboeiro e Quixelô). Para isto, foram realizadas visitas de campo nos municípios, entrevistas com os gestores responsáveis pela gestão dos resíduos sólidos e consultas às publicações disponíveis, à legislação e normativos. A partir dos resultados obtidos, verificou-se que as condições dos sistemas de gestão integrada dos resíduos sólidos dos municípios estudados foram classificadas como Gestão Inadequada, tendo o não atendimento à diretrizes estabelecidas pela Política Nacional de Resíduos Sólidos e as inadequadas condições operacionais como os principais fatores que comprometeram a qualidade dos referidos sistemas. verificou-se que os desafios ainda são grandes, no que se refere a coleta seletiva, apenas o município de Jucás implementou parcialmente este tipo de coleta. Também foi constatado que nenhum dos municípios pesquisados possuía uma política pública voltada para a avaliação das potencialidades e das fragilidades dos seus sistemas de gestão de resíduos sólidos, o que dificultava o diagnóstico por parte dos gestores e, em consequência, o planejamento e execução das intervenções públicas mais urgentes.
\end{abstract}

Palavras-chave: Gestão municipal; Indicadores de sustentabilidade; Planos municipais; Política Nacional de Resíduos Sólidos.

\begin{abstract}
The aim of this study is to evaluate the condition of integrated solid waste management in municipalities in the Centro-Sul region of Ceará, using the Updated Waste Management Condition Index (ICGRA). This index was developed with 62 indicators arranged in four items: system characteristics, system planning, operational conditions and management alignment with the National Solid Waste Policy and new techniques. The methodological approach was carried out by applying the ICGRA spreadsheet to each of the municipalities studied (Iguatu, Jucás, Cariús, Saboeiro and Quixelô). For this, field visits were carried out in the municipalities, interviews with managers responsible for solid waste management and consultations to available publications, legislation and regulations. From the results obtained, it was found that the conditions of the integrated solid waste management systems of the municipalities studied were classified as Inadequate Management, with non-compliance with the guidelines established by the National Solid Waste Policy and inadequate operating conditions as the main factors that compromised the quality of these systems. it was found that the challenges are still great, with regard to selective collection, only the municipality of Jucás partially implemented this type of collection. It was also found that none of the municipalities surveyed had a public policy aimed at assessing the strengths and weaknesses of their solid waste management systems, which made it difficult for managers to diagnose and, consequently, to plan and implement interventions most urgent public services.
\end{abstract}

Keywords: Municipal management; Sustainability indicators; Municipal plans; National Solid Waste Policy.

\section{Resumen}

El objetivo de este estudio es evaluar el estado de la gestión integrada de residuos sólidos en los municipios de la región Centro-Sul de Ceará, utilizando el Índice de Condición de Gestión de Residuos Actualizado (ICGRA). Este índice se desarrolló con 62 indicadores ordenados en cuatro ítems: características del sistema, planificación del sistema, condiciones operativas y alineación de la gestión con la Política Nacional de Residuos Sólidos y nuevas 
técnicas. El abordaje metodológico se realizó aplicando la hoja de cálculo ICGRA a cada uno de los municipios estudiados (Iguatu, Jucás, Cariús, Saboeiro y Quixelô). Para ello, se realizaron visitas de campo en los municipios, entrevistas con los responsables de la gestión de residuos sólidos y consultas a las publicaciones disponibles, legislación y normativa. A partir de los resultados obtenidos, se encontró que las condiciones de los sistemas integrados de gestión de residuos sólidos de los municipios estudiados se clasificaron como Gestión Inadecuada, siendo el incumplimiento de los lineamientos establecidos por la Política Nacional de Residuos Sólidos y las condiciones operativas inadecuadas como principales factores. que comprometió la calidad de estos sistemas. Se encontró que los desafíos aún son grandes, en cuanto a la recolección selectiva, solo el municipio de Jucás implementó parcialmente este tipo de recolección. También se encontró que ninguno de los municipios encuestados contaba con una política pública orientada a evaluar las fortalezas y debilidades de sus sistemas de gestión de residuos sólidos, lo que dificultaba a los gestores diagnosticar y, en consecuencia, planificar e implementar las intervenciones de los servicios públicos más urgentes.

Palabras clave: Gestión municipal; Indicadores de sostenibilidad; Planes municipales; Política Nacional de Residuos Sólidos.

\section{Introdução}

O desenvolvimento econômico, o crescimento populacional, a urbanização e a revolução tecnológica vêm sendo acompanhados por alterações no estilo de vida e nos modos de produção e consumo da população. Como decorrência direta desses processos, vem ocorrendo um aumento na produção de resíduos sólidos, tanto em quantidade como em diversidade, principalmente nos grandes centros urbanos. Além do acréscimo na quantidade, os resíduos produzidos passaram a abrigar em sua composição elementos sintéticos e perigosos aos ecossistemas e à saúde humana, em virtude das novas tecnologias incorporadas ao cotidiano (Fricke et al., 2015; Gouveia, 2012).

Na Gestão Integrada de Resíduos, as ações são direcionadas no sentido de contribuírem para a sustentabilidade ambiental, econômica e social. Por causa de seu foco na flexibilidade e especificidade para as condições locais, esta forma de gestão não prescreve soluções. Mantém princípios que permitem que as localidades desenvolvam seus próprios sistemas, sempre na busca de soluções para o uso racional dos recursos ambientais, ao combate a todas as formas de desperdício e à minimização da geração de resíduos.

No Brasil, a gestão dos resíduos sólidos é de competência principal dos municípios, haja vista o artigo 30, inc. I e V, da Constituição Federal (Brasil, 1988), bem como o artigo 10 da Lei 12.305/2010 (Brasil, 2010), incumbindo aos entes municipais a gestão dos resíduos sólidos gerados nos respectivos territórios. Um dos instrumentos legais da Política Nacional de Resíduos Sólidos (PNRS), instituída pela da Lei 12.305/2010, é o Plano Municipal de Gestão Integrada de Resíduos Sólidos (PMGIRS).

A PNRS prevê que os municípios que optarem por soluções consorciadas terão prioridade no acesso aos recursos da União. (Brasil, 2010). No entanto, os municípios são responsáveis pela própria gestão de resíduos, enquanto os consórcios intermunicipais têm por objetivo a elevação das escalas de aproveitamento e redução dos custos envolvidos, sendo dever do Estado apoiar e priorizar as iniciativas do município de soluções consorciadas ou compartilhadas entre dois ou mais municípios.

Nesse sentido, uma importante ferramenta para os gestores públicos e os órgãos de controle e de fiscalização obterem informações sobre a condição dos sistemas de gestão integrada de resíduos sólidos é a utilização de sistemas de indicadores. Estes, por sua vez, refletem a diversidade de fatores que influenciam no desempenho dos referidos sistemas. Indicadores referem-se a dados capazes de apontar e fornecer informações sobre uma situação, permitindo avaliações atuais e possibilidades futuras, como também comparações entre fatos e lugares. Os indicadores são um complexo de dados que, organizados de forma simplificada, são capazes de dispor informações sobre o que se está analisando (Landim, 2020). As informações geradas a partir destes indicadores contribuem para a tomada de decisão em relação ao que fazer, para que fazer, como fazer, com quem e com o que fazer em sistemas de gestão (Campani, 2012). 
$\mathrm{Na}$ literatura, são encontrados trabalhos que utilizaram, por exemplo, índices para avaliarem as condições de sustentabilidade da coleta seletiva e das organizações dos catadores (Bringhenti et al., 2011; Besen, 2017), bem como a condição de gestão de resíduos sólidos (Dantas, 2008; Mendez, 2017; Olay- Romero et al. 2020; Silva et al. 2019). O Índice da Condição de Gestão de Resíduos Atualizado (ICGRA) aplicado nesse estudo é composto por meio de 62 indicadores dispostos em quatro categorias: características do sistema, planejamento do sistema, condições operacionais e alinhamento da gestão à Política Nacional de Resíduos Sólidos e às modernas técnicas de manejo dos resíduos.

Esse estudo tem como objetivo é avaliar a condição da gestão integrada de resíduos nos municípios de Iguatu, Quixelô, Cariús, Jucás e Saboeiro da região do Sertão Centro-Sul, no estado do Ceará, aplicando ICGRA, visando elencar suas fortalezas e fragilidades a fim de propor melhorias nas práticas e políticas públicas voltadas para esta matéria, priorizando as intervenções públicas mais urgentes. Os municípios pertencem ao Consórcio Regional de Resíduos Sólidos do Alto Jaguaribe (CORRAJ), este consórcio visa exercer, em escala regional, as atividades de planejamento dos serviços públicos de limpeza urbana e manejo dos resíduos no território dos municípios consorciados.

\section{Metodologia}

A amostra de estudo foram os municípios de Iguatu, Jucás, Quixelô, Cariús e Saboeiro, localizados na região Sertão Centro Sul do Estado do Ceará (Figura 1).

Figura 1 - Municípios que compõem a área de estudo na Região Centro Sul.

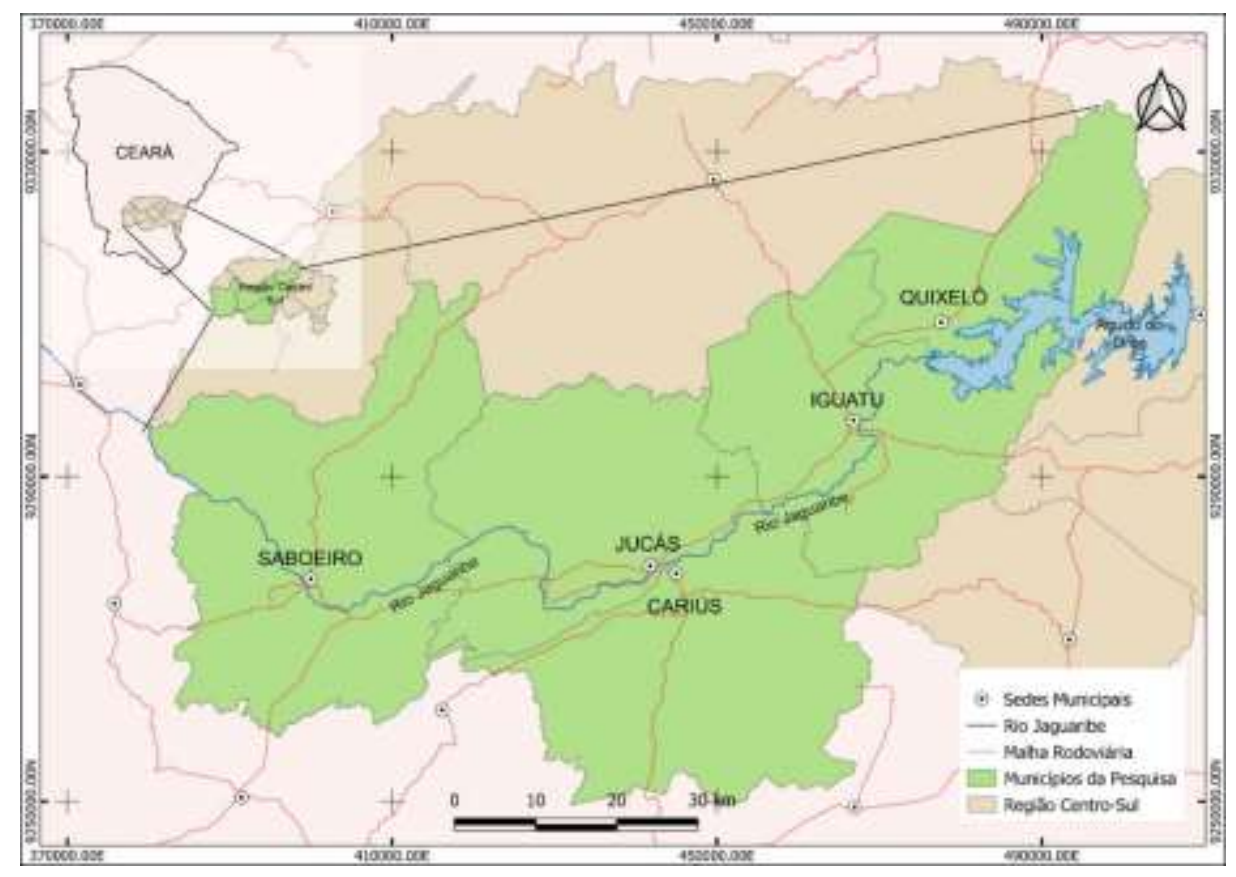

Fonte: Autores (2021).

A pesquisa foi tratada de forma descritiva, exploratória e documental, de natureza qualitativa e quantitativa, delineada por pesquisa bibliográfica.

Para o desenvolvimento desta pesquisa foram coletados dados primários referentes às formas e condições de gerenciamento dos resíduos sólidos nas Secretarias dos municípios estudados e por meio de pesquisa de campo, onde foram averiguadas as características reais dos sistemas de gestão dos municípios estudados. Durante a visita aos municípios, foram realizadas entrevistas com os gestores municipais, responsáveis pela gestão de resíduos sólidos, para obtenção de dados referentes aos indicadores considerados nas planilhas do ICGRA. 
De acordo com Gil (2008), a característica descritiva consiste em analisar e descrever com profundidade algum fato ou fenômeno, havendo a recomendação da utilização de informações obtidas através de estudos exploratórios. A pesquisa exploratória objetiva dar explicações sobre um fato cujo tema é pouco explorado, demandando levantamento bibliográfico, análise de documentos e observações de fatos. A investigação bibliográfica consiste na análise de documentos de domínio científico como livros, periódicos, artigos científicos e outros, enquanto a documental busca informações em documentos que ainda não receberam tratamento científico (Gil,1987; Marconi e Lakatos, 2012). A abordagem quantitativa será utilizada nos resultados da entrevista semiestruturada que será aplicada aos gestores municipais. A análise qualitativa ser utilizada à interpretação, explicação e significação das informações obtidas no que tange a gestão dos resíduos (Gil, 2007).

Na Tabela 1 consta a população, a área territorial e a densidade populacional de cada município considerado nesta pesquisa.

Tabela 1 - Amostra do estudo de caso.

\begin{tabular}{cccc}
\hline Municípios & População (Hab.) & Área Territorial $\left(\mathbf{k m}^{2}\right)$ & Densidade Demográfica $\left(\mathbf{h a b} . / \mathbf{~ k m}^{2}\right)$ \\
\hline Iguatu & 103.633 & 992.208 & 93,76 \\
Jucás & 24.949 & 940.336 & 25,40 \\
Quixelô & 16.116 & 605,345 & 26,81 \\
Cariús & 18.700 & 1.036 .417 & 17,49 \\
Saboeiro & 15.757 & 1.381 .274 & 11,39 \\
\hline
\end{tabular}

Fonte: IBGE (2021).

Para a determinação do índice de condição de gestão de resíduos sólidos foi utilizada a metodologia de Méndez (2017), que corresponde a uma atualização da metodologia de Dantas (2008), uma vez que foram acrescentados, no cálculo deste índice, indicadores relacionados as diretrizes, instrumentos e ferramentas estabelecidas pela PNRS. Na composição do ICGRA, são considerados 40 indicadores relacionados as características do sistema, planejamento do sistema e condições operacionais, e 22 indicadores relacionados as diretrizes, instrumentos e ferramentas propostas pela PNRS. O enquadramento final do desempenho dos municípios varia de 0 a 10 nas seguintes faixas: de 0 a 7,9 para a gestão inadequada e 8,0 a 10,0 para a gestão adequada.

\section{Resultados e Discussão}

Para obter a avalição final e medir a situação da gestão, a planilha do ICGRA foi aplicada considerando indicadores relacionados aos itens Característica do Sistema (CS), Planejamento do Sistema (PS), Condições Operacionais (CO) e Adequação a PNRS (AP). Para que a condição de gestão dos municípios seja classificada como adequada, o resultado do ICGRA precisa ser igual ou superior a 8,0.

A maior pontuação do ICGRA foi obtida para o município de Jucás $(6,2)$. Os outros quatro municípios obtiveram pontuação abaixo de 5,5. De maneira geral verificou-se a seguinte ordem decrescente de classificação da condição de gestão dos municípios: Jucás, Saboeiro, Iguatu, Cariús e Quixelô.

Os resultados do ICGRA (Figura 2), mostra que a condição de gestão dos RS foi classificada inadequada em todos os municípios estudados, uma vez que os valores do ICGRA foram inferiores a 8. 
Figura 2 - Resultado final do ICGRA nos municípios.

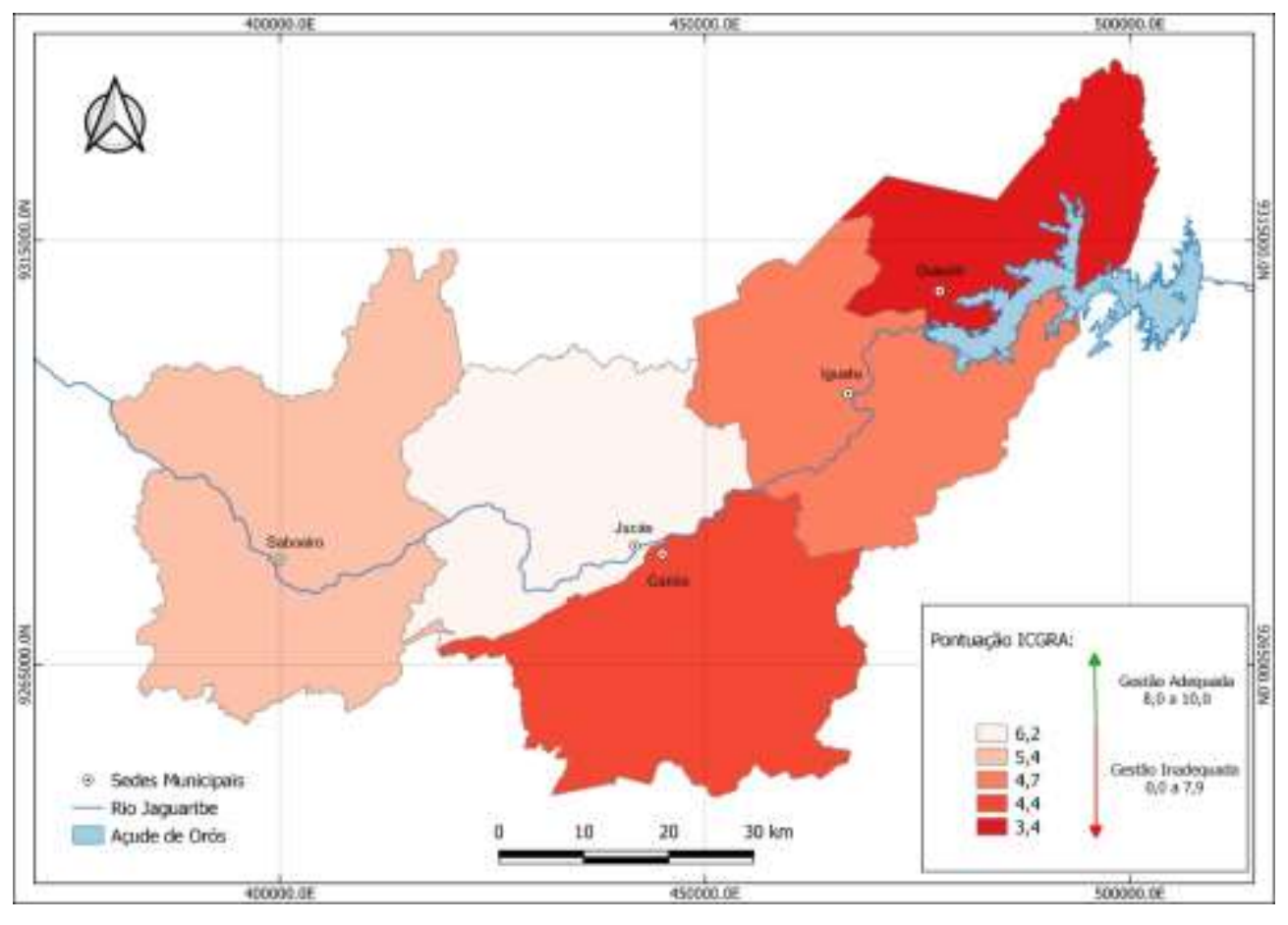

Fonte: Autores (2021).

Dantas (2008) e Queiroga (2010), ao aplicarem o ICGR em municípios do estado do Rio de Janeiro e da Paraíba, respectivamente, bem como Méndez (2017) e Souto (2019), ao aplicarem o ICGRA em municípios do estado do Rio de Janeiro e do Rio grande do Norte, nesta devida ordem, obtiveram resultados que classificaram a condição de gestão como inadequada.

Ao se fazer uma análise da pontuação dos itens avaliados no cálculo do ICGRA de todos os municípios, verificou-se que o menor desempenho foi obtido no item Adequação a Política Nacional de Resíduos Sólidos (AP), seguido do item Condições Operacionais (CO). Na sequência vem as Características do Sistema (CS), exceto para os municípios Saboeiro e Jucás, e Planejamento do Sistema (PS).

$\mathrm{Na}$ Tabela 2 constam os percentuais relacionados a pontuação de cada item avaliado no ICGRA dos municípios pesquisados, além do resultado de cada índice e a condição de gestão dos RS de cada município.

Tabela 2 - Percentual da pontuação obtida para cada item considerado no cálculo do ICGRA dos municípios estudados.

\begin{tabular}{|c|c|c|c|c|c|c|c|c|c|c|}
\hline \multirow{2}{*}{ Município } & \multicolumn{2}{|l|}{ CS } & \multicolumn{2}{|l|}{ PS } & \multicolumn{2}{|l|}{$\mathrm{CO}$} & \multicolumn{2}{|l|}{$\mathbf{A P}$} & \multirow{2}{*}{ ICGRA } & \multirow{2}{*}{ Avaliação } \\
\hline & Pontos & $(\%)$ & Pontos & $(\%)$ & Pontos & $(\%)$ & Pontos & $(\%)$ & & \\
\hline Saboeiro & 20 & 62,5 & 28 & 73,6 & 35 & 58,3 & 28 & 35,8 & 5,4 & Gestão Inadequada \\
\hline Jucás & 26 & 87,5 & 30 & 78,9 & 45 & 75 & 25 & 34,2 & 6,2 & Gestão Inadequada \\
\hline Cariús & 12 & 46,8 & 24 & 63,1 & 28 & 46,6 & 26 & 35,6 & 4,4 & Gestão Inadequada \\
\hline Quixelô & 13 & 50 & 20 & 52,6 & 29 & 48,3 & 8 & 10,2 & 3,4 & Gestão Inadequada \\
\hline Iguatu & 20 & 62,5 & 33 & 86,8 & 28 & 46,6 & 15 & 20,5 & 4,7 & Gestão Inadequada \\
\hline $\begin{array}{l}\text { Pontuação } \\
\text { Máxima }\end{array}$ & 32 & --- & 38 & --- & 60 & --- & 73 & --- & --- & --- \\
\hline
\end{tabular}


Observa-se na CS que o percentual máximo referente a pontuação deste item foi de 87,5\%, o qual foi obtido no município de Jucás. Iguatu e Saboeiro pontuaram com 62,5\% e os municípios de Quixelô e de Cariús com menos de 50\%. É importante destacar que os municípios de Quixelô e Cariús possuem população menor que 20 mil habitantes. Este resultado é atribuído ao baixo percentual de coleta regular domiciliar e comercial (inferior a $70 \%$ ) e a inexistência de coleta de resíduos do serviço de saúde constatados nestes municípios. Para um melhor desempenho de um sistema de gestão de RSU eficiente, é necessário investimentos em projetos que conduzam a redução, a coleta seletiva e a reciclagem, resultando em benefícios a população e ao meio ambiente (Barbosa e Carvalho Júnior, 2020).

Em relação ao PS, os municípios de Iguatu, Jucás e Saboeiro apresentaram os maiores percentuais relacionados as pontuações deste item: Iguatu com 86,8 \%, Jucás com 78,9 \% e Saboeiro com 73,6 \%. Os municípios de Cariús e Quixelô apresentaram os menores percentuais, com $63,1 \%$ e 52,6 \% na devida ordem. Os menores desempenhos destes municípios neste item se devem a inexistência do plano integrado de gestão de resíduos da construção civil e do planejamento da gestão de resíduos de serviços de saúde, comuns aos dois municípios em questão. Também contribuíram negativamente para a pontuação do item PS nesses municípios a inexistência do controle de solicitações e reclamações, bem como das equipes de fiscalização e de programas de educação ambiental e conscientização.

Ante o exposto é importante enfatizar que, educação ambiental é mediadora da apropriação, pelos sujeitos, das qualidades, capacidades e desenvolvimento individual de um caráter social em sua relação com a natureza e com os outros seres humanos. Ela é um fator imprescindível ao gerenciamento adequado e sustentável dos resíduos sólidos sendo utilizada como instrumento para a reflexão das pessoas no processo de mudança de atitudes em relação ao correto manejo dos resíduos e à valorização do meio ambiente. (Peneluc e Silva, 2008; Gusmão, 2000).

Salienta-se, ainda, que o planejamento se trata de uma etapa fundamental na gestão para identificar como está o nível de satisfação com o sistema atual e alcançar a sustentabilidade dos resíduos sólidos, sendo essencial para todas as etapas da gestão de resíduos, desde a concepção e planejamento até a operação e descarte (Tsai et al. 2021).

Os indicadores que elevaram a pontuação do item PS na maioria dos municípios estudados foram o apoio a gestão participativa e consórcios e os programas de inclusão de catadores no sistema. A participação da população na gestão dos RS dos municípios pesquisados contribui à democratização das informações e para que a sociedade civil atue como importante ator social nos processos de decisão dos serviços associados ao manejo de resíduos sólidos prestados pelas gestões municipais. Com relação aos consórcios, a adesão dos municípios a este tipo de instrumento favorece a redução de custos e viabiliza a solução conjunta do problema, principalmente nos municípios de menor porte (Quixelô, Saboeiro, Cariús e Jucás).

Quanto aos programas de inclusão de catadores no sistema, salienta-se que a organização dos catadores na forma de associação (Iguatu, Quixelô e Jucás) ou em associações de moradores (Cariús) deveria propiciar condições favoráveis à prática da coleta seletiva e para a inclusão socioeconômica dessa população. Contudo, foi verificado que apenas o município de Jucás realizava a coleta seletiva, mesmo que parcialmente. Esta constatação indica que apesar da existência de programas de inclusão de catadores nos 4 municípios supracitados, ainda existe um caminho muito longo a ser percorrido para que os catadores possam usufruir dos benefícios associados a atividade de coleta de material reciclável.

Na Figura 3 é ilustrada a situação de cada município em relação a pontuação obtida em cada um dos itens avaliados no ICGRA. 
Figura 3 - Resultados dos municípios por itens avaliados do ICGRA.

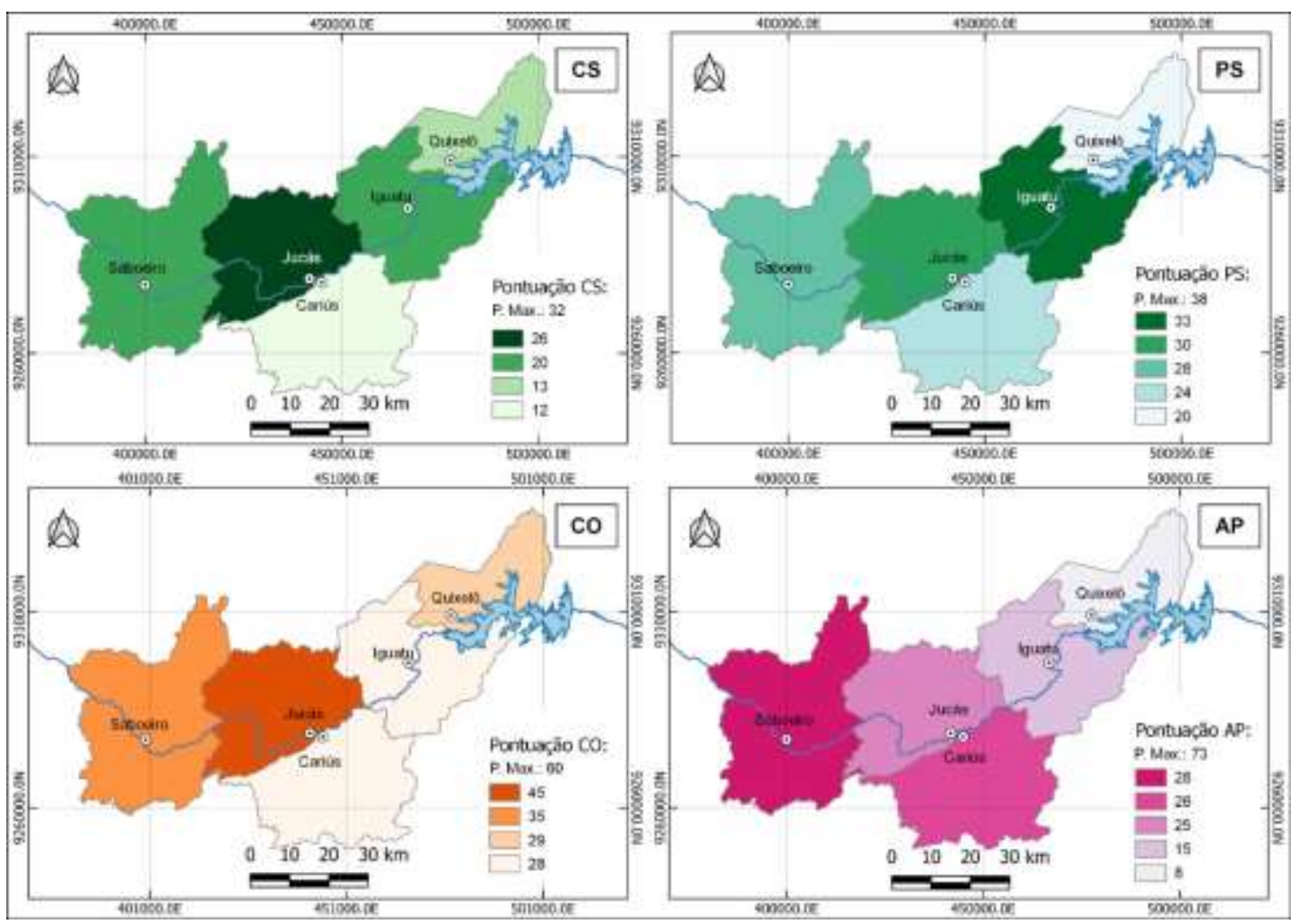

Fonte: Autores (2021).

Em relação as Condições Operacionais (CO), foi verificado que, de maneira geral, as menores pontuações foram obtidas nos municípios de Iguatu, Cariús e Quixelô (Figura 3). Os municípios que apresentaram pontuações mais próximas ao valor máximo deste item foi Jucás e Saboeiro.

Depreende-se que os indicadores que mais contribuíram para a diminuição da pontuação do item CO na maioria dos municípios pesquisados foram: destinação final de RSS, controle de desempenho, avaliação crítica, operação de unidade de reciclagem, reaproveitamento dos resíduos orgânicos, destinação final dos resíduos sólidos urbanos do município, controle de acidentes do trabalho e controle de absenteísmo nas equipes.

O controle dos aspectos que interferem no desempenho dos serviços de limpeza pública e manejo dos resíduos sólidos estão relacionados, de acordo com Dantas (2008), ao controle das quantidades de resíduos coletadas e enviadas para tratamento ou disposição final. No entanto, podem existir pequenas ações de controle, como fiscalização da qualidade dos serviços executados. Estas devem ser documentadas para que seja possível uma avaliação por meio da identificação dos resultados auferidos, alinhados com os objetivos traçados. Seguindo esse contexto, as avaliações críticas são necessárias para introduzir melhorias no sistema de gestão, identificando os problemas de operação do dia a dia, permitindo, assim, serem realizadas medidas corretivas, objetivos e metas.

Observa-se que o reaproveitamento dos resíduos orgânicos na maioria dos municípios é inexistente, sendo enviados para lixões. O descarte inadequado desses resíduos possui um alto potencial de contaminação ao liberar gases de efeito estufa e chorume $^{1}$ por meio da sua decomposição (Siqueira e Abreu, 2016). Outro ponto pertinente é a segurança dos trabalhadores que realizam a limpeza dos grandes centros urbanos. Vale ressaltar o quanto é importante haver um controle da sua segurança no trabalho a fim de evitar possíveis prejuízos físicos, sociais e econômicos, sendo, portanto, necessário investir em ações

\footnotetext{
${ }^{1}$ Líquido resultante da infiltração de águas pluviais no maciço de resíduos e da água que constitui os resíduos orgânicos liberada durante sua decomposição leva a degradação.
} 
mitigadoras no controle para reduzir acidentes no ambiente de trabalho garantindo a saúde dos trabalhadores (Mosciatti, 2018). Esse fato também é importante no contexto do absenteísmo nas equipes de trabalho, uma vez que é necessário que os serviços sejam realizados com regularidade. A esse respeito Dantas (2008) enfatiza a importância da implementação de medidas de controle do absenteísmo nas equipes de limpeza pública para que funcionários faltosos não prejudiquem a execução de serviços como varrição, coleta e outros.

No que se refere a avaliação dos indicadores relacionados ao atendimento as diretrizes da PNRS e as novas técnicas para a gestão de resíduos sólidos (AP), verificou-se que a pontuação deste item variou de 8,0 a 28,0 (Figura 3). Este resultado mostra que nenhum dos municípios pesquisados atingiu nem metade do valor máximo desse item da planilha do ICGRA, que é de 73 pontos.

Verificou-se que os municípios não atendem aos diferentes requisitos da PNRS, assim como, questões relacionadas a novas técnicas dos processos de gestão de resíduos. Tal fato, confirma a pouca adesão às inovações da PNRS, estando muito longínquo de temas como manejo dos resíduos sólidos, regulamentação da logística reversa, monitoramento ambiental, aproveitamento de biogás e destinação final adequada dentre outros. De maneira geral observa-se que os indicadores Remoção de materiais inservíveis como móveis e outros e Preenchimento das informações do Sistema Nacional de Informações de Resíduos (SNIS) pontuaram positivamente em todos os municípios pesquisados. O pleno atendimento do primeiro indicador a PNRS está associado ao fato da coleta dos materiais inservíveis não ser uma atividade regular, sendo, portanto, realizada apenas quando solicicitada pela polpulação, o que facilita bastante a execução deste serviço. Quanto ao segundo indicador citado, a ação que o mesmo representa é uma condição indispensável para que os municípios que pertencem ao CORRAJ recebam recursos financeiros de arrecadações do ICMS ecológico para serem gastos na gestão dos resíduos sólidos urbanos. Ou seja, é um incentivo fiscal instituído pela Lei $\mathrm{n}^{\circ}$ 12.305/2010 que induz a comportamentos favoráveis à gestão integrada e ao gerenciamento ambientalmente adequado dos resíduos sólidos urbanos.

Dentre os indicadores que contribuíram desfavoravelmente para a pontuação do item AP, são destacados: o plano de contingência para greve de funcionários dos serviços de limpeza urbana, o sistema de controle da frota por GPS ou SIG, o monitoramento geotécnico ambiental nas áreas de disposição irregular de resíduos desativadas, a operação de triagem de resíduos licenciados e funcionando corretamente, a coleta ou aproveitamento do biogás gerado nas áreas de disposição final de resíduos, disponibilização de coletor adequado para o armazenamento pré-coleta, o sistema de gestão implantado (ISO 9.000 ou 14.001), incentivos economicos para a não geração, redução, reutilização e reciclagem, contrato de monitoramento geotécnico e ambiental dos aterros sanitáios e contratação de seguro de responsabilidade civil para os geradores de resíduos perigosos (art. 40 PNRS).

A ausência, na maioria dos municípios estudados (a exceção é o município de Cariús), do plano de contingência para greve de funcionários dos serviços de limpeza urbana implica no fato de que em situações de paralização na execução destes serviços pode ocorrer o acúmulo de resíduos nas via públicas. Este acúmulo pode causar poluição ambiental e comprometer a saúde pública, desvalorizar imóveis e obstruir vias públicas, prejudicando o trânsito de pedestres, ciclistas e até de veículos, além de reduzir o escoamento das águas superficiais e, em consequência, causar alagamentos. Salienta-se que o plano de contingência referido acima consta no PRGIRS no qual estão inseridos os municípios estudados nesta pesquisa. As medidas preventivas para contingências podem ser adotadas no âmbito das ações administrativas, como manter cadastro de empresas fornecedoras dos serviços para contratação em caráter emergencial (Ceará,2016).

No que se refere ao Sistema de controle da frota por Geoprocessamento para o tratamento da informação geográfica/Global Positioning System (GPS) ou Sistemas de Informações Geográficas (SIG), sua ausência traz prejuízos financeiros e ambientais, uma vez que os serviços de operação de coleta absorvem consideravelmente uma boa parte dos recursos municipais destinados à limpeza urbana. 
Os serviços de limpeza absorvem entre 5 e $15 \%$ dos recursos de um orçamento municipal, dos quais 40 a $60 \%$ são destinados exclusivamente à coleta e ao transporte de resíduos (Vilhena, 2018). Assim, para ter um percurso que atenda as restrições de movimentação dos veículos coletores de lixo nas vias das cidades, com o menor custo, aproveitando a máxima capacidade dos caminhões e o melhor tempo de serviço é preciso definir um conjunto de rotas que atendam um conjunto de determinadas áreas (Lacerda, 2003). Por isso, rotas definidas por meios de estudos e planejamentos acabam por evitar transtornos além de ajudar na diminuição de gastos (Apaydin \& Gonullu, 2007; Vilhena, 2018).

Com relação aos indicadores monitoramento geotécnico e ambiental nas áreas de disposição irregular de resíduos desativadas, coleta ou aproveitamento do biogás gerado nas áreas de disposição final de resíduos e contrato de monitoramento geotécnico e ambiental dos aterros sanitáios, verificou-se que todos os municípios pontuaram negativamente nestes quesitos. Contudo, ressalta-se que nenhum deles possuía aterro sanitário e apenas o município de Jucás possuía área de lixão desativada, mas não realizava o monitoramento geotécnico e ambiental da mesma.

De acordo com o Caderno técnico de reabilitação de áreas degradadas por resíduos sólidos urbanos (Feam, 2010), o processo de recuperação ambiental dessas áreas deve envolver técnicas de desativação, adequação provisória como aterro controlado e recuperação como aterro sanitário. A esse respeito é importante salientar que áreas de lixões desativados e não encerrada de e forma adequada podem causar poluição do solo e da água superficial e subterrânea, além de riscos de acidentes geológico-geotécnicos (Jesus, 2020).

No que se refere a operação de triagem, apenas o município de Jucás recebeu pontuação. Nos demais municípios ainda não tinham sido implantado o serviço de coleta seletiva. Segundo Ferri et al (2015), os centros de triagem possuem um papel importante, pois permitem a separação dos materiais que são passíveis de serem reciclados e contribuem com a redução no custo de transporte dos resíduos até o aterro.

A avaliação dos indicadores disponibilização de coletor adequado para o armazenamento pré-coleta e incentivos econômicos para a não geração, redução, reutilização e reciclagem mostra que a maioria dos municípios estudados precisam implementar ações eficazes voltadas para o atender aos princípios básicos da política de pré-aterro.

Seguindo esse contexto, o sistema de gestão ISO 9.000 ou 14.001 ainda é visto como um grande desafio na gestão dos resíduos (prefeitura, empresa pública ou concessionária). Todos os municípios avaliados mostraram não possuir algum tipo de certificação na modalidade ISO, quer seja na área da qualidade (9.001) ou na área da gestão ambiental (14.001). Esta última segue a metodologia conhecida por aplicar um ciclo de melhoria contínua chamado Plan-Do-Check-Act (PDCA), que significa Planejar-Executar-Verificar-Agir, conduzindo, portanto, a ações direcionadas para a obtenção de avanços para garantir o crescimento organizacional. Contudo, esses sistemas são difíceis de serem implementados em municípios de pequeno porte (Souto, 2019; Santana et al, 2018).

No que tange a contratação de seguro de responsabilidade civil para os geradores de resíduos perigosos estabelebilicido no art. 40 da PNRS, foi observada a baixa pontuação dos municípios nesse indicador. No entanto, é importante frizar que o licenciamento ambiental de empreendimentos ou atividades que trabalhem com resíduos perigosos está atrelada a contratação de seguro de responsabilidade civil por danos causados ao meio ambiente ou à saúde pública, observando sempre as regras sobre cobertura e os limites máximos de contratação fixados em regulamentos por meio do órgão licenciador (Brasil, 2010). Este seguro se subdivide em seguro de responsabilidade civil quando cobre o pagamento de indenização por perdas e danos materiais e/ou corporais devidos pelo segurado a terceiros e seguro garantia, quando se destina a assegurar ao segurado o cumprimento de uma obrigação pelo tomador do seguro. O pagamento poderá ser direto ao terceiro prejudicado ou o reembolso do segurado que arcou previamente com a indenização, a critério de cada contrato (Custodio \& Massonetto,2021). 
O baixo desempenho as adequações da PNRS dos municípios estudados não é uma realidade isolada dos municípios pesquisados. Moraes (2017), ao avaliar os processos de elaboração, implantação e implementação dos planos de gestão integrada de resíduos sólidos nos municípios da região Centro Sul do estado do Ceará, também identificou que a maioria dos municípios considerados em seu estudo não contemplavam os requisitos e exigências mínimas estabelecidos pela PNRS.

De acordo com Pimenta et al. (2020), dentre as maiores dificuldades enfrentadas por muitos municípios brasileiros para se adequarem as diretrizes e novas técnicas da PNRS, está a falta de repasse dos recursos financeiros para este fim. Neste sentido, IPT (2018) e Queiroga (2010), relatam que o baixo desempenho dos sistemas de gestão integrada de resíduos sólidos urbanos, principalmente nos pequenos municípios do Brasil, é atribuído ao fato de que a maior parte dos recursos financeiros para financiamento do setor provém dos municípios onde há muitas dificuldades para a arrecadação.

Porém, em relação aos recursos financeiros destinados a gestão de resíduos sólidos dos municípios pesquisados, é importante destacar que estes municípios participam, desde o ano 2019, por meio do CORRAJ, da seleção do Índice Municipal de Qualidade do Meio Ambiente (IQM). Este índice possibilita que os municípios recebam recursos financeiros oriundos de arrecadações do Imposto sobre Circulação de Mercadorias e Serviços (ICMS) ecológico ${ }^{2}$, para serem gastos na gestão dos resíduos sólidos urbanos. Porém, o valor do recurso a ser recebido depende da pontuação obtida por cada município no IQM. De acordo com dados do CORRAJ, todos os municípios que fazem parte deste consórcio obtiveram a pontuação máxima (1) no IQM desde junho de 2019 e o repasse dos recursos passou a ser feito desde janeiro de 2020. No ano de 2021, no período de janeiro a julho, o valor recebido por cada município foi de R \$213.267,11 (Ceará, 2021).

\section{Conclusão}

A partir da investigação da condição da gestão integrada dos resíduos sólidos dos municípios de Iguatu, Quixelô, Cariús, Jucás e Saboeiro, localizados na região do Sertão Centro-Sul, no estado do Ceará, aplicando o Índice da Condição de Gestão de Resíduos Atualizado (ICGRA), foram obtidas as seguintes conclusões:

- As condições dos sistemas de gestão integrada dos resíduos sólidos dos municípios estudados foram classificadas como inadequadas e se apresentaram na seguinte ordem decrescente de classificação: Jucás, Saboeiro, Iguatu, Cariús e Quixelô. Destaca-se que a variação da condição da gestão dos resíduos sólidos entre os referidos municípios não estava associada ao quantitativo de recursos financeiros disponibilizados para tal fim, já que todos receberam o mesmo valor do repasse do ICMS ecológico, mas sim da capacidade técnica das instituições e profissionais envolvidos na prestação dos serviços de limpeza urbana e manejo de resíduos sólidos de cada município.

- Dentre as quatro categorias de indicadores avaliados no ICGRA (CS, PS, CO e AP) de cada município investigado, a que mais comprometeu o valor do referido índice foi a que se referia a adequação a Política Nacional de Resíduos Sólidos e Novas Técnicas, seguida do item Condições Operacionais. Esse resultado enfatiza a baixa adesão dos responsáveis pela gestão dos resíduos sólidos ao cumprimento das diretrizes estabelecidas pela PNRS e a desarticulação entre o que foi planejado e o que foi executado no âmbito da operacionalização dos sistemas de gestão.

- A implementação da coleta seletiva nos municípios pesquisados, apesar de ser um procedimento essencial para o bom desempenho da gestão do RSU e, portanto, fundamental para se alcançar os objetivos de disposição final correta dos resíduos, ainda é uma realidade bastante desafiadora na área estudada, uma vez que apenas o município de Jucás realizava este tipo de atividade de forma parcial concentrando-se $\mathrm{n}$ apenas na zona urbana.

Propõe-se em estudos futuros, um aprofundamento para a definição de métodos de ponderação de indicadores; estimar e detalhar o grau de importância de cada indicador no índice final de avaliação, através do risco do não atendimento de

\footnotetext{
${ }^{2}$ ICMS ecológico: é um mecanismo tributário que possibilita aos municípios acesso a parcelas maiores que àquelas que já têm direito, dos recursos financeiros arrecadados pelos Estados através do Imposto sobre Circulação de Mercadorias e Serviços.
} 
determinado indicador; realizar um acompanhamento da evolução da condição sustentável e ambiental das áreas estudadas utilizando-se os mesmos indicadores considerados nesta pesquisa para efeito de comparação.

\section{Agradecimentos}

Ao Programa de Pós-Graduação em Desenvolvimento Regional Sustentável (PRODER), da Universidade Federal do Ceará no Cariri (UFCA) Campus Crato, por possibilitar momentos de estudos e reflexão rumo ao desenvolvimento sustentável, aos gestores dos municípios Iguatu, Quixelô, Cariús, Jucás e Saboeiro pela participação e disponibilidade para que esta pesquisa fosse realizada.

\section{Referências}

Apaydin, O., \& Gonullu, M. T. (2007). Route optimization for solid waste collection: Trabzon (Turkey) case study. Global NEST Journal, 9(1), 6-11.

Barbosa, L. N. V., \& de Carvalho Júnior, F. H. (2020). Estudo de indicadores para a gestão de resíduos sólidos urbanos no município de MaracanaúCeará. Revista Meio Ambiente e Sustentabilidade, 9(18).

Besen, G. R., Günther, W. M. R., Ribeiro, H., Jacobi, P. R., \& Dias, S. M. (2017). Gestão da coleta seletiva e de organizações de catadores: indicadores e índices de sustentabilidade. São Paulo: Faculdade de Saúde Pública/USP.

Brasil. (2010) Palácio do Planalto. LEI No 12.305 Institui a Política Nacional de Resíduos Sólidos; altera a Lei no 9.605 , de 12 de fevereiro de 1998; e dá outras providências. http://www.planalto.gov.br/ccivil_03/_ato2007-2010/2010/lei/112305.htm.

Brasil. Constituição (1988). Constituição da República Federativa do Brasil. Brasília, DF: Senado Federal. http://www.planalto.gov.br/ ccivil_03/constituicao/constituicao.htm

Bringhenti, J. R., Zandonade, E., \& Günther, W. M. R. (2011). Selection and validation of indicators for programs selective collection evaluation with social inclusion. Resources, Conservation and Recycling, 55(11), 876-884.

Campani, D. B. (2012). Indicadores socioambientais como instrumento de gestão na coleta seletiva de resíduos sólidos urbanos. Dissertação de mestrado Instituto de hidráulicas, Universidade Federal do Rio Grande do Sul.

Custodio, V. M., \& Massonetto, L. F. (2021). A exigibilidade de seguro de responsabilidade civil por dano ambiental como condicionante do licenciamento ambiental brasileiro. Revista de Direito da Cidade, 13(1), 130-159.

da Conceição Peneluc, M., \& Silva, S. A. H. (2008). Educação ambiental aplicada à gestão de resíduos sólidos: análise física e das representações sociais. Revista Entreideias: educação, cultura e sociedade, 13(14). http://arquivos.ambiente.sp.gov.br/cea/2016/08/R3.pdf.

Dantas, K. M. C. (2008) Proposição e avaliação de sistemas de gestão ambiental integrada de resíduos sólidos através de indicadores em municípios do Estado do Rio de Janeiro. Tese (Doutorado) - Programa de Pós-Graduação de Engenharia, Universidade Federal do Rio de Janeiro, Rio de Janeiro.

dos Santos Severino, J., Vries, P., Santana, D., \& Amarante, M. (2018). Sistema de Gestão Integrado ISO 9001, 14001 e OHSAS 18001. Revista Pesquisa e Ação, 4(1), 192-208.

Ferri, G. L., Chaves, G. D. L. D., \& Ribeiro, G. M. (2015). Analysis and location of urban solid waste collection/inspection centers for a reverse logistics network: a case study in São Mateus-ES. Production, 25(1), 27-42.

Fricke, K. (Ed.). (2015). Gestão sustentável de resíduos sólidos urbanos: transferência de experiência entre a Alemanha e o Brasil. Techn. Univ.

Fundação Estadual Do Meio Ambiente - FEAM. (2010). Caderno técnico de reabilitação de áreas degradadas por resíduos sólidos urbanos. Fundação Israel Pinheiro. Belo Horizonte. https://israelpinheiro.org.br/wp-content/uploads/2016/09/Caderno-T\%C3\%A9cnico-de-Reabilita\%C3\%A7\%C3\%A3o-de\%C3\%81reas-Degradadas-por-Res\%C3\%ADduos-S\%C3\%B3lidos-Urbanos.pdf. Acesso em 25 de setembro de 2021.

GIL, A. C. (2007). Como elaborar projetos de pesquisa. (4a ed.), Atlas.

GIL, A. C. (2008). Métodos e técnicas de pesquisa social. (6a ed.), Atlas.

GIL. A.C. (1987). Métodos e técnica de pesquisa social. (2a ed.), Atlas.

Gouveia, N. (2012). Resíduos sólidos urbanos: impactos socioambientais e perspectiva de manejo sustentável com inclusão social. Ciência \& saúde coletiva, $17,1503-1510$.

Gusmão, O. S. (2000). Reciclagem artesanal na UEFS: estratégia educacional na valorização do meio ambiente. In Congresso Nacional de Meio Ambiente na Bahia. 2, 56-58.

Instituto Brasileiro De Geografia E Estatística. (IBGE). (2021). Cidades e Estados. https://www.ibge.gov.br/cidades-e-estados/ce/saboeiro.html 
Research, Society and Development, v. 10, n. 14, e365101422026, 2021

(CC BY 4.0) | ISSN 2525-3409 | DOI: http://dx.doi.org/10.33448/rsd-v10i14.22026

Jesus, A. O. C. (2020) Avaliação preliminar da poluição do solo e da água subterrânea da área de um lixão desativado. https://www.bdtd.uerj.br:8443/bitstream/1/16615/2/Disserta\%c3\%a7\%c3\%a3o\%20-\%20Andressa\%20Oliveira\%20Costa\%20de\%20Jesus\%20-\%202020\%20$\% 20$ Completo.pdf.

Lacerda, M. G. (2003). Análise de uso de SIG no sistema de coleta de resíduos sólidos domiciliares em uma cidade de pequeno porte. 145 p. Dissertação (mestrado) - Universidade Estadual Paulista. Faculdade de Engenharia de Ilha Solteira. Área de concentração: Recursos Hídricos e Tecnologias Ambientais. Ilha Solteira. https://repositorio.unesp.br/handle/11449/98108

Landim, G. H. P, Brito. M. G. S. L, \& Nunes, F. C. B. (2020). Índice de Esgotamento Sanitário em Área de Vulnerabilidade Social de Juazeiro do Norte-CE sob a Ótica do Desenvolvimento Sustentável. Juazeiro do Norte, CE: Quipá Editora.114 p. http://deposita.ibict.br/handle/deposita/138

Marconi, M. D. A.; \& Lakatos, E. M. (2012). Metodologia do Trabalho Científico. (7a ed.), Atlas. 225 p.

Mendéz, G. P. (2017). Avaliação da gestão municipal de resíduos sólidos através de indicadores ambientais (Dissertação de mestrado). Universidade Federal do Rio de Janeiro, https://pantheon.ufrj.br/handle/11422/9766\#: :text=O\%20presente\%20estudo\%20compreendeu\%20a,s\%C 3\%B3lidos\% 20atrav\%C3\%A9s\%20de\%20indicadores\%20ambientais.\&text=A\%20metodologia\%20proposta\%20mostrou\%20ser,\%2F\%20IQA\%2F\%20IQS\%20e\%20ICG $\mathrm{R}$.

Moraes, J. L. D. (2017). Os planos municipais de gestão integrada de resíduos sólidos e sua implantação em municípios da Região Centro Sul do Ceará. $126 \mathrm{f}$. Tese (doutorado) - Universidade Estadual Paulista, Instituto de Geociências e Ciências Exatas. Rio Claro.

Mosciatti, J. V. D. (2018). Redução no número de acidentes no serviço de coleta de resíduos sólidos urbanos. Curitiba. http://repositorio.utfpr.edu.br/jspui/bitstream/1/18317/1/CT_CEEST_XXXVI_2018_22.pdf.

Nascimento, L. L. O (2017). Avaliação comparativa entre a política nacional de resíduos sólidos e a política municipal de resíduos da cidade de Campos dos Goytacazes. Boletim do Observatório Ambiental, Campos dos Goytacazes/RJ, 11(2), 19-30.

Olay-Romero, E., Turcott-Cervantes, D. E., del Consuelo Hernández-Berriel, M., de Cortázar, A. L. G., Cuartas-Hernández, M., \& de la Rosa-Gómez, I. (2020). Technical indicators to improve municipal solid waste management in developing countries: A case in Mexico. Waste Management, 107, 201-210.

Pimenta, S. S., Costa, D. S., Silva, E. R. A. C., \& Arouche-Lima, I. M. (2020). Análise da gestão e gerenciamento de resíduos sólidos urbanos em Alcântara (Maranhão-Brasil). Meio Ambiente (Brasil), 2(1).

Queiroga, E. C. D. (2010). Adequabilidade da utilização do índice de condição da gestão de resíduos ICGR para municípios do Estado da Paraíba. $75 \mathrm{f}$. Dissertação (Mestrado em Engenharia Urbana e Ambiental) Universidade Federal da Paraíba, João Pessoa. https://repositorio.ufpb.br/jspui/handle/tede/5571.

Silva, M. N., \& Siqueira, V. L. (2017). Riscos ocupacionais de catadores de materiais recicláveis: ações em saúde e segurança do trabalho. Revista Osvaldo Cruz.[internet].

Souto, V. S. A. (2019). Avaliação da gestão de resíduos sólidos em Municípios do Seridó. 137 f. Dissertação (Mestrado Profissional em Uso Sustentável de Recursos Naturais) - Instituto Federal de Educação, Ciência e Tecnologia do Rio Grande do Norte, Natal, 2019. https://memoria.ifrn.edu.br/handle/1044/1702?show=full

Tsai, F. M., Bui, T. D., Tseng, M. L., Lim, M. K., Wu, K. J., \& Mashud, A. H. M. (2021). Assessing a hierarchical sustainable solid waste management structure with qualitative information: policy and regulations drive social impacts and stakeholder participation. Resources, Conservation and Recycling, 168 , 105285.https://doi.org/10.1016/j.resconrec.2020.105285

Vilhena, A. (2018). Lixo Municipal. Manual de Gerenciamento Integrado, 3, 241-252. 